\title{
Search efficiency is not sufficient: The nature of search modulates stimulus-driven attention
}

\author{
Koeun Jung ${ }^{1} \cdot$ Suk Won $\operatorname{Han}^{1} \cdot$ Yoonki Min ${ }^{1}$
}

Published online: 1 October 2018

(C) The Psychonomic Society, Inc. 2018

\begin{abstract}
It has long been debated whether or not a salient stimulus automatically attracts people's attention in visual search. Recent findings showed that a salient stimulus is likely to capture attention especially when the search process was inefficient due to high levels of competition between the target and distractors. Expanding these studies, the present study proposes that a specific nature of visual search, as well as search efficiency, determines whether or not a salient, task-irrelevant singleton stimulus captures attention. To test this proposition, we conducted three experiments, in which participants performed two visual search tasks whose underlying mechanisms are known to be different: orientation-feature search and Landolt-C search tasks. We found that color singleton distractors captured attention when participants performed the orientation-feature search task. The magnitude of this capture effect increased as search efficiency decreased. On the contrary, the capture by singleton distractors was not observed under the Landolt-C search task. This differential pattern of capture effect was not due to differences in search efficiency across the search tasks; even when search efficiency was controlled for, stimulus-driven capture of attention by a salient distractor was found only under the feature search. Based on these results, the present study suggests that in addition to search efficiency, the nature of search strategy and the extent to which attentional control is strained play crucial roles in observing stimulus-driven attentional capture in visual search.
\end{abstract}

Keywords Attentional capture $\cdot$ Singleton distractor $\cdot$ Search mechanism $\cdot$ Attentional control

It has long been recognized that a novel, salient stimulus captures people's attention (Pavlov, 1927; Sokolov, 1963). This stimulus-driven attentional capture has also been known to take place when orienting toward the salient stimulus hampers the concurrent goal-directed behavior. This is well illustrated by studies using the additional singleton paradigm. In a classic study (Theeuwes, 1992), participants searched for the target stimulus (a circle) among multiple distractors (diamonds). Importantly, in some trials, a singleton distractor (a distractor

Suk Won Han and Yoonki Min contributed equally to this work.

Electronic supplementary material The online version of this article (https://doi.org/10.3758/s13414-018-1598-4) contains supplementary material, which is available to authorized users.

Suk Won Han

suk.w.han@gmail.com

Yoonki Min

ykmin@cnu.ac.kr

1 Department of Psychology, Chungnam National University, Daejeon, Korea with a unique color) was presented (singleton distractor present), while in others, no such singleton was presented. As a result, search reaction time was significantly longer for the singleton distractor-present trials than for the singleton distractor-absent trials. This result has been interpreted as that the color singleton distractor captures attention in a stimulusdriven manner, interfering with the target search.

However, other studies found that salient, singleton stimuli do not always capture attention. A seminal study by Bacon and Egeth (1994) demonstrated that salient stimuli capture attention only when they match the observer's goal-driven task set. According to the goal-driven account of attentional capture, under the task setting developed by Theeuwes (1992), participants might have adopted the strategy of detecting any singleton stimulus to perform the task as the target stimulus was an item with a unique shape (shape singleton). Under this "singleton search mode," participants' attention is likely to be captured by any kind of singleton stimulus, such as a color singleton. To test this hypothesis, Bacon and Egeth created a search task, in which the singleton detection strategy is not applicable; the target stimulus, a circle, was surrounded by a 
set of heterogenous distractors, comprising diamonds, a square, and a triangle. In this case, participants should search for stimuli containing specific target features. Under this feature search mode, a task-irrelevant color singleton distractor, which did not contain any target feature, did not capture attention (see also Lamy \& Egeth, 2003).

In response, it has recently proposed that the size of attentional window plays an important role for observing attentional capture by a salient, goal-irrelevant stimulus. Specifically, Theeuwes (2004) argued that stimulus-driven attentional capture should be observed only when search was highly efficient, such that search performance was not affected by the number of search items. Notably, under this efficient search, the attentional window should be set wide. On the contrary, when the search was inefficient, yielding a significant set-size effect, the size of attentional window is reduced, thereby the salient stimulus falling outside the attentional window. In this case, no capture should be found. That is, stimulus-driven attentional capture takes places when increasing search set size does not increase search reaction time, obviating the need to serially shift attention among search stimuli. However, Leber and Egeth (2006) challenged this claim by showing that the adoption of feature search mode eliminates attentional capture even when the search was efficient.

While this debate has not been completely resolved, some researchers recently reported findings that salient stimuli, which are entirely irrelevant with the current task goal, capture attention. Specifically, in the study by Barras and Kerzel (2017b), participants searched for a square either among diamonds (inefficient search) or circles (efficient search). Critically, in half of the trials, one of the distractors had a distinct color from the rest (color singleton distractor). Given that the target was defined by shape, this singleton distractor did not match the top-down attentional set. Remarkably, this singleton distractor captured attention both when the search was efficient and inefficient. Another important finding of this study is that the magnitude of stimulus-driven attentional capture increased as search efficiency decreased, challenging the claim by Theeuwes (2004).

The modulation of attentional capture by search efficiency is also supported by recent neuroscienfic evidence (Barras and Kerzel, 2017a). In the difficult visual search of this study, an $\mathrm{N} 2 \mathrm{pc}$ component of the event-related potential (ERP) occurred to the color singleton distractor. The result indicates attentional capture because the N2pc component is a negative deflection of the ERP at posterior electrodes contralateral to the position where focal attention is allocated. On the contrary, with easy visual search, a distractor positivity (Pd) component to the color distractor occurred, which means attentional suppresstion. In line with this, another group also showed that stimulus-driven attentional capture is better detected when the search is inefficient (Gaspelin, Ruthruff, Lien, \& Jung, 2012; Gaspelin, Ruthruff, \& Lien, 2016).
These studies provide clear evidence that a salient stimulus, irrelevant with the top-down task set, can capture attention and that this capture increases as the efficiency of the concurrent search increases. Based upon these findings, search efficiency was suggested to be a crucial factor to observe stimulus-driven attentional capture. Indeed, these findings are in line with a large number of studies showing that the effect of stimulusdriven attention becomes pronounced as competition between stimuli increases (Beck \& Kastner, 2005, 2009; Desimone \& Duncan, 1995; Han \& Marois, 2014; Kastner, De Weerd, Desimone, \& Ungerleider, 1998).

Notably, the studies (Barras and Kerzel, 2017b; Gaspelin et al., 2012; Gaspelin et al., 2016) challenging the attentional window account posit that large set-size effect is associated with serial search, while a small or nonsignificant set-size effect reflects parallel search. However, while the absence of set-size effect clearly indicates that search proceeded in a parallel manner, robust and significant set-size effect does not necessarily indicate that the search is performed in a serial manner; limited parallel search, in which capacity-limited attentional resource is divided into multiple items, can produce a similar magnitude of a set-size effect as serial search predicts (Sung, 2008). Furthermore, recent studies showed that the performance of some visual search tasks yielding robust setsize effect is not dependent on capacity-limited attentional processes. Specifically, even though a given search can be done without the involvement of attentional control, the presence of statistical decision noise can produce significant setsize effects (Han, 2017; Huang \& Pashler, 2005; Mazyar, Van den Berg, \& Ma, 2012; McElree \& Carrasco, 1999; Treisman $\&$ Gelade, 1980).

Given this, it has yet to be clarified whether the pattern of attentional capture by a singleton distractor would be different, depending on whether a given search is done in a serial or a parallel manner. To address this issue, we examined the attentional capture effect by a singleton distractor in visual search, employing two different visual search tasks whose underlying processes are known to be different: orientationfeature search and Landolt-C search tasks. The Landolt-C search task is well known to heavily strain attentional control; this search requires the multiple shifting of attention in a serial manner (Woodman \& Luck, 1999, 2003, 2007). It is also commonly agreed that this search task consumes capacitylimited attentional resources. Even though some feature searches can be as inefficient as the Landolt- $\mathrm{C}$ search, this does not mean that the feature search is performed in a similar manner to that of the Landolt-C search. Palmer and his colleagues provided evidence that difficult feature-based searches lead to significant search slopes due to decision noise (Palmer, 1994; Palmer, Ames, \& Lindsey, 1993; Palmer, Verghese, \& Pavel, 2000; Palmer \& Wright, 1998).

Given the purpose of the present study, the efficiencies of these two search tasks should be matched. To achive this, for 
the orientation-feature search, in which participants searched for a tilted line among vertical lines, the search efficiency was manipulated by varying the magnitude of the target tilt (Proulx \& Egeth, 2006). For the Landolt-C search task, in which the target was a square with a gap on the left or right side with the distractors being top-gap or bottom-gap squares, the search efficiency was manipulated by varing the gap size of the squares. Having controlled for difference in search efficieny across different search tasks, we tested the effect of a singelton distractor on search performance during the feature and Landolt-C searches.

To predict results, if search efficiency solely determines the size of the attentional window and the strength of attentional capture by a singleton stimulus, then two tasks having similar search efficiencies should yield a comparable magnitude of attentional capture by a salient stimulus. Alternatively, the magnitude of attentional capture might differ depending on the nature of search processes. Specifically, the capture effect might be more pronounced under the feature search than under the Landolt-C search because the latter taxes attentional control to a great extent. With the implementation of highlevel attentional control, the likelyhood that attention is oriented toward a salient but task-irrelevant stimulus would decrease, or stimulus-driven orienting might be inhibited.

\section{Experiment 1}

\section{Method}

\section{Participants}

Two groups of 12 volunteers (ages: 20-29 years; 20 female) participated for course credit or monetary compensation. A group of participants performed the Landolt-C search task, while the other performed the orientation-feature search task. For the Landolt-C search task, the sample size was estimated based on a previous study by Woodman, Luck, and Schall (2007), in which 10 participants performed the Landolt-C search task, yielding significant set-size effects. For the orientation-feature search task, we ran a power analysis based on a data set from our pilot study $(N=7)$. This analysis revealed that a sample size of 12 should be enough to detect a significant capture effect by a singleton distractor at the power level of .80 when the tilt of the target is $9^{\circ}$. Given these, we decided to use the sample size of 12 .

All participants gave informed consent and had normal color vision and normal or corrected-to-normal visual acuity. All experimental procedures were approved by the Chungnam University Institutional Review Board and were performed in accordance with the approved guidelines.

\section{Stimuli and apparatus}

The experiment was programmed and run using PsychoPy (Peirce, 2007). The stimuli were presented on a 21 -in. LCD monitor with a black background. Viewing distance was set to about $60 \mathrm{~cm}$.

In the feature search task, the search display had five or nine lines, whose length was $1.0^{\circ}$ of visual angle. The target was a line tilted to the right or the left, while distractors were vertical lines (see Fig. 1). The tilt of the target was either $4.5^{\circ}$, $9^{\circ}, 13.5^{\circ}$, or $18^{\circ}$. In the Landolt-C search task, the search display contained five or nine outlined squares $\left(1.5^{\circ} \times 1.5^{\circ}\right)$ with a gap. The target had the gap either on the left or right side of the square, while the distractors had the gap either on the top or bottom side of the square. The gap size was set to $0.3^{\circ}, 0.6^{\circ}, 0.9^{\circ}$, or $1.2^{\circ}$.

A half of the total trials had a distinct color distractor, which are referred to as distractor-present trials, while the other half had no singleton distractor (distractor-absent trials). For each distractor-present trial, the color of the singleton distractor was randomly selected from a pool of five colors (red, yellow, green, orange, and cyan) to increase interference effects compared with a constant distractor color (Barras and Kerzel, 2017b; Kerzel \& Barras, 2016). The search items were presented on an imaginary circle with a radius of $8.0^{\circ}$.

\section{Procedure and design}

Each trial began with the 500-ms presentation of a fixation dot, followed by the presentation of the search display that remained until participants made a response (within 5,000 $\mathrm{ms}$ ). For the orientation-feature search task, participants were told to search for a right-tilted or left-tilted line among vertical lines and to report the orientation of the tilted line. For the Landolt-C search task, participants looked for an outlined square with a right or left gap among squares with a top or bottom gap, and were required to indicate whether the gap was on the left or right. Participants were instructed to respond as accurately and quickly as possible. Importantly, they were informed that stimuli with distinct colors are always distractors and paying attention to those would interfere with the search process.

Taken together, the experiment consisted of a $2 \times 2 \times 2 \times 4$ mixed design with singleton distractor (present vs. absent), set size (five vs. nine), and search difficulty (four levels depending on gap size or target tilt) as within-subjects factors and search task as a between-subjects factor. For each group, participants performed four blocks, each of which included 256 trials (feature search task) or 216 trials (Landolt-C search). Set size varied within block, whereas search difficulty level varied between blocks. The block order was randomized across participants. Prior to the main experimental session, each 
(a)

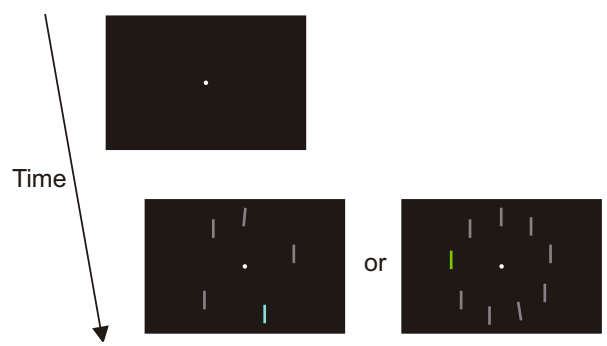

Fig. 1 Examples of experimental trials. a Orientation-feature search task. b Landolt-C search task. In both search tasks, singleton distractors were present on half of all trials, which are referred to as distractor-present

participant performed 10 32 practice trials to become familiar with the task.

\section{Results and discussion}

The results of Experiment 1 are shown in Fig. 2 and Table 1. To analyze visual search reaction time (RT) data, only trials with correct search responses were used. A mixed-factor analysis of variance (ANOVA) with singleton distractor, set size, and search difficulty as within-subjects factors and search task as a between-subjects factor was applied to the RT data. The full details of the ANOVA results are presented in Table S1 in the Supplementary Material. Given the significant three-way interaction between search task, search difficulty, and singleton distractor, $p=.011$, we compared the capture effect by a (b)

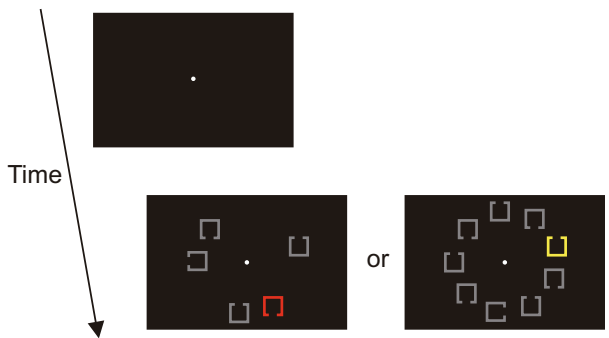

trials. In the other half, the search displays had no singleton distractor (distractor-absent trials). (Color figure online)

singleton distractor across search difficulty for each search task.

For the feature search group, an ANOVA with singleton distractor, set size, and search difficulty as factors revealed significant main effects of search difficulty, $F(3,33)=$ 226.3, $p<.001, \eta^{2}=.95$; set size, $F(1,11)=253.4, p<$ $.001, \eta^{2}=.96 ;$; and singleton distractor, $F(1,11)=7.408, p$ $=.02, \eta^{2}=.40$. The interaction between set size and search difficulty was significant, $F(3,33)=166.3, p<.001, \eta^{2}=.94$. This significant interaction indicates that our manipulation of search difficulty successfully affected search efficiency.

Importantly, the interaction between search difficulty and singleton distractor was also significant, $F(3,33)=8.141, p<$ $.001, \eta^{2}=.43$, showing that the capture effect of the singleton distractor did differ across the search difficulty levels. To further clarify under which condition the capture effect of the
Orientation feature search

(a)

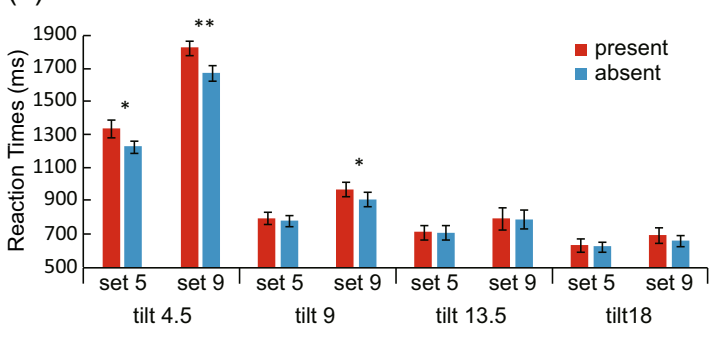

Landolt-C search

(c)

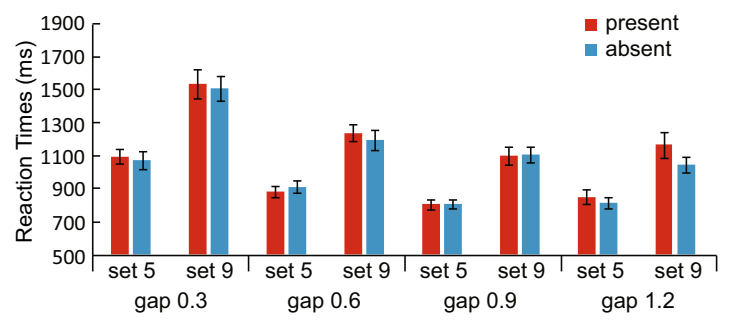

(b)

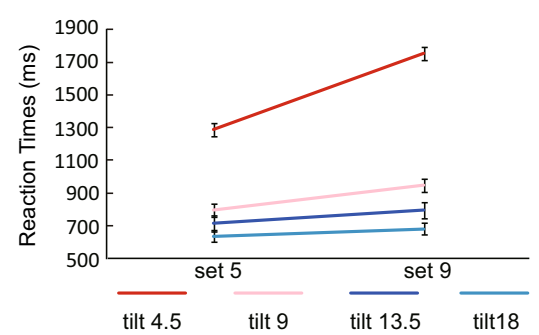

(d)

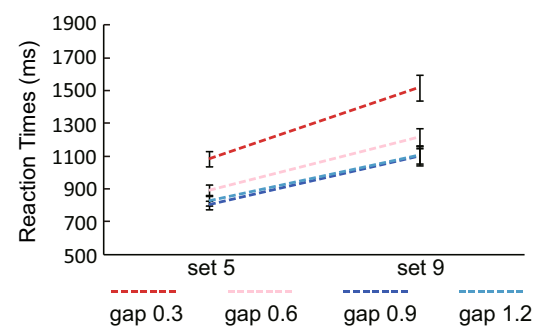

Fig. 2 Search RTs and search slopes of Experiment 1. Error bars represent standard errors of the means. Mean reaction times of orientation-feature search task (a) and Landolt-C search task (c). Search slopes for each search difficulty levels under orientation-feature search task (b) and Landolt-C search task (d). Search slope calculation included distractor-present and distractor-absent conditions 
Table 1 Mean accuracy data for Experiment 1

Tasks and search difficulty Singleton distractor

\begin{tabular}{lllll} 
& Present & Absent & Present & Absent \\
\hline Orientation-feature search & Set 5 & & Set 9 & \\
Tilt 4.5 & $97.26 \%$ & $98.44 \%$ & $95.57 \%$ & $97.14 \%$ \\
Tilt 9 & $99.48 \%$ & $98.31 \%$ & $98.57 \%$ & $98.44 \%$ \\
Tilt 13.5 & $97.52 \%$ & $98.44 \%$ & $97.79 \%$ & $98.70 \%$ \\
Tilt 18 & $97.66 \%$ & $97.40 \%$ & $98.57 \%$ & $97.53 \%$ \\
Landolt-C search & Set 5 & & Set 9 & \\
Gap 0.3 & $99.23 \%$ & $100 \%$ & $98.92 \%$ & $98.15 \%$ \\
Gap 0.6 & $99.54 \%$ & $99.38 \%$ & $99.38 \%$ & $99.69 \%$ \\
Gap 0.9 & $99.69 \%$ & $99.07 \%$ & $99.07 \%$ & $98.77 \%$ \\
Gap 1.2 & $99.54 \%$ & $99.38 \%$ & $99.54 \%$ & $99.23 \%$ \\
\hline
\end{tabular}

singleton distractor was found, we ran pair-wise $t$ tests. These $t$ tests revealed that with the tilt of $4.5^{\circ}$, the presence of a singleton distractor significantly slowed search response when the set size was nine (151 ms, 1,833 vs. 1,682) and the set size was five $(110 \mathrm{~ms}, 1,343$ vs. 1,232$), t \mathrm{~s}(11)>2.36, \mathrm{ps}<$ .038 . When the tilt of the target was $9^{\circ}$, the capture of attention by the singleton distractor was found only when the set size was nine (59 ms, 975 vs. 916$), t(11)=2.363, p=.0375$. No capture effect was found under other conditions ( $p s>.20)$. These results indicate that the stimulus-driven attentional capture by a salient stimulus was more pronounced when search efficiency was low than when the search was relatively efficient. This is a replication of the previous studies (Barras and Kerzel, 2017b; Gaspelin et al., 2016).

For the Landolt-C search group, main effects of search difficulty, $F(3,33)=30.28, p<.001, \eta^{2}=.73$, and set size, $F(1,11)=188.3, p<.001, \eta^{2}=.94$, were significant. The interaction between search difficulty and set size was also significant, $F(3,33)=9.379, p<.001, \eta^{2}=.46$. This interaction also indicates that the set-size effect significantly varied as a function of search difficulty. Importantly, contrary to the result of the orientation-feature search group, the singleton distractor did not interfere with target search $(p>.25)$.

The same ANOVA applied to the accuracy data. The full details of the ANOVA results are presented in Table S2 in the Supplementary Material. The resulting mean accuracy dates are shown in Table 1. These data show that the current RT results were not contaminated by a speed-accuracy trade-off.

Taken together, the results of Experiment 1 showed that under feature search, a color singleton distractor captured attention when the search was highly inefficient (Levels 1, 2). This is a consistent finding with the previous study showing that the capture effect by a salient singleton distractor increased as search efficiency decreased (Barras and Kerzel, 2017b). Further, these results are relevant to recent findings by Barras and Kerzel (2017a). They showed that there was attentional capture by the color distractor in inefficient search, whereas there was suppression to the distractor in efficient search. By contrast, attentional capture by the salient singleton distractor was not found at any difficulty level under the Landolt-C search. Importantly, this differential pattern of results is not due to difference in search efficiency across the search tasks; the search efficiencies (slopes) of the most difficult level of the two search tasks were not significantly different $(p>.46)$, but the attentional capture by the singleton stimulus of the two tasks showed contrasting patterns. If search efficiency had been a sole determinant for observing stimulusdriven attentional capture, then capture effect by the singleton distractor should also have been found under the Landolt-C search.

\section{Experiment 2}

Experiment 2 was performed to further confirm the role of search mechanisms in stimulus-driven attentional capture. Given that significant attentional capture by a singleton distractor was pronounced under feature search when the set size was 9, only this set size was included in the present experiment. In addition, we included only the two most inefficient search conditions for both the feature search and Landolt-C search. Another notable difference from Experiment 1 was that all the independent variables, singleton distractor (present vs. absent), search difficulty (easy vs. difficult), and search task (feature vs. Landolt C) were randomly intermixed within blocks.

\section{Method}

\section{Participants}

Twenty-one new volunteers (ages 20-29 years; 10 female) participated for course credit or monetary compensation and gave informed consent. All participants had normal color vision and normal or corrected-to-normal visual acuity. One participant's search performance was poor (error rates $>20 \%$ ), and was excluded from further analysis. The final sample consisted of 20 participants. The sample size was determined on the basis of the data from Experiment $1(N=24)$, in which participants performed the feature or Landolt-C search task (with set size nine) in the presence or absence of a singleton distractor. A power analysis using this data set revealed that a sample size of 18 should be sufficient to detect a significant interaction effect between the experimental factors (search task, search difficulty, and singleton distractor) at the level of .90. Given this, we decided to collect data from 20 participants. All experimental procedures were approved by the Chungnam University Institutional Review Board and were performed in accordance with the approved guidelines. 


\section{Stimuli and apparatus}

Stimuli and apparatus were identical to those of Experiment 1, except for the following. First, search display always had nine search items. Second, for each search task, we had two different levels of search difficulty (easy vs. difficult). For the orientation-feature search task, the tilt of the target was either 4.5 (difficult) or $9^{\circ}$ (easy). For the Landolt-C search, the size of gap was set to either 0.3 (difficult) or $0.6^{\circ}$ (easy).

\section{Procedure and design}

The procedure and design were identical to those of Experiment 1, with the following exceptions. In Experiment 2 , search task varied within participants. Thus, participants could not predict whether the task would be a feature search task or Landolt-C search. Hence, the experiment consisted of a within-subjects $2 \times 2 \times 2$ design with singleton distractor (present vs. absent), search difficulty (difficult vs. easy), and search task (feature search vs. Landolt-C search) as factors. Participants performed six experimental blocks, each of which contained 144 trials.

\section{Results}

To analyze visual search reaction time (RT) data, only trials with correct search responses were used. A repeated-measures three-way ANOVA with singleton-distractor, search difficulty, and search task was applied to the RT data. Mean RTs are shown in Fig. 3. The RT analysis revealed significant main effects of search task, $F(1,19)=27.8, p<.001, \eta^{2}=.59$, and search difficulty, $F(1,19)=316.1, p<.001, \eta^{2}=.94$. While the main effect of singleton distractor was not observed, $F(1$, 19) $=2.276, p=.15$, the interaction between singleton distractor and search task was significant, $F(1,19)=11.33$, $p=.003, \eta^{2}=.37$. The two-way interaction between search task and search difficulty was also significant, $F(1,19)=$ 252.3, $p<.001, \eta^{2}=.93$. Finally, the interaction between search difficulty and singleton distractor was also significant $F(1,19)=10.22, p=.005, \eta^{2}=.35$. The three-way interaction was not significant $(p>.25)$.

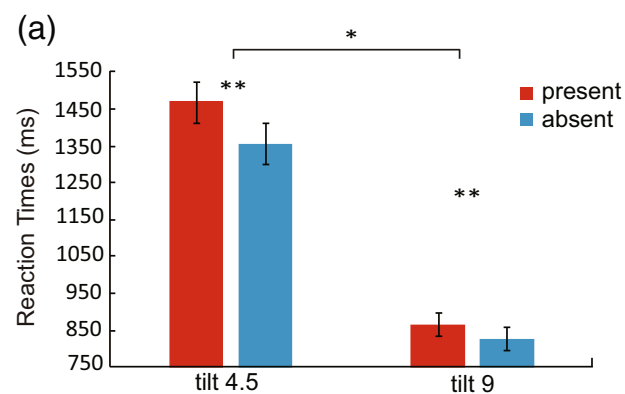

Notably, despite the lack of significant three-way interaction, we separately examined the feature search and the Landolt-C search data due to the following two reasons. First, previous studies (e.g., Barras and Kerzel, 2017b) and our Experiment 1 commonly showed that the capture effect is modulated by search efficiency under feature search. These previous findings justify the current analyses. Second, the two-way interaction between search task and singleton distractor was significant, necessitating the examination of capture effects in different tasks.

For both search tasks data, we applied a two-way ANOVA with singleton distractor and search difficulty as factors. In line with the result of Experiment 1, under feature search task, significant attentional capture was found; the main effect of singleton distractor was significant, $F(1,19)=17.61, p<.001$, $\eta^{2}=.48$. The interaction between singleton distractor and search difficulty was also significant, $F(1,19)=7.78, p=$ $.012, \eta^{2}=.29$. This interaction indicates that the capture effect with the target tilt of $4.5^{\circ}(114 \mathrm{~ms}, p<.002)$ was significantly greater than the capture effect with the target tilt of $9^{\circ}(38 \mathrm{~ms}$, $p<.003)$. Under the Landolt-C search task, neither the main effects nor interaction related to singleton distractor was significant $(p s>.4)$.

The three-way ANOVA was also applied to the accuracy data. The full details of the ANOVA results are presented in Table S3 in the Supplementary Material. The resulting accuracy rates are shown in Table 2. Most importantly, search accuracy was high with no difference between the distractorpresent and distractor-bsent trials $(p>.4)$. These results show that the current RT results were not contaminated by a speedaccuracy trade-off. The results of Experiment 2 are clear. The attentional capture by a color singleton distractor was observed under the orientation-feature search whereas it was not found under the Landolt-C search, replicating Experiment 1. Further, the capture under feature search conditions significantly increased when the search efficiency decreased, consistent with previous findings by Barras and Kerzel (2017b). Importantly, both the easy and difficult Landolt-C search tasks yielded a more robust set-size effect than the easy feature search. If search efficiency was the primary factor responsible for observing significant attentional

(b)

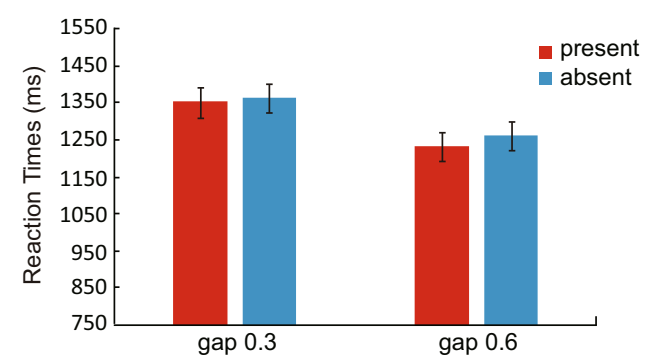

Fig. 3 Search RTs of Experiment 2. Error bars represent standard errors of the means. Mean reaction times of orientation-feature search task (a) and Landolt-C search task (b) 
Table 2 . Mean accuracy data for Experiments 2 and 3

Singleton distractor

\begin{tabular}{llll}
\hline $\begin{array}{l}\text { Present } \\
\text { Experiment 2 }\end{array}$ & Absent & $\begin{array}{l}\text { Present } \\
\text { Experiment 3 } 3\end{array}$ & Absent \\
& &
\end{tabular}

Orientation-feature search

$\begin{array}{lllll}\text { Tilt } 4.5 & 97.36 \% & 97.45 \% & 94.44 \% & 96.71 \%\end{array}$

$\begin{array}{lllll}\text { Tilt } 9 & 98.24 \% & 98.75 \% & 97.54 \% & 97.64 \%\end{array}$

Landolt-C search

$\begin{array}{lllll}\text { Gap } 0.3 & 99.58 \% & 99.21 \% & 99.17 \% & 99.03 \% \\ \text { Gap 0.6 } & 99.49 \% & 99.67 \% & 99.21 \% & 99.26 \%\end{array}$

capture by a salient stimulus, we should have found the capture effect under the Landolt-C search. These results suggest that search efficiency cannot fully explain the capture of attention by a singleton distractor.

\section{Experiment 3}

One might argue that attentional capture under the Landolt- $\mathrm{C}$ search is absent simply because this search task included heterogeneous distractors (top-gap or bottom-gap stimuli), whereas a homogeneous set of distractors (vertical lines) were used in the feature search. Indeed, Bacon and Egeth (1994) found that color singleton distractors did not capture attention in the presence of heterogeneous distractors. Experiment 3 was performed to address this issue.

\section{Method}

\section{Participants}

Twenty-three new volunteers (ages 20-29 years; 11 female) participated for course credit or monetary compensation and gave informed consent. All participants had normal color vision and normal or corrected-to-normal visual acuity. Three participant's search performances were poor (error rates $>20 \%$ ) and were excluded from further analysis. The final sample consisted of 20 participants.

\section{Stimuli, apparatus, procedure, and design}

The experimental design and stimuli were identical to Experiment 2, with the following exception. Participants performed the Landolt-C search task with homogenous distractors. The Landolt-C search task had search displays in which a set of distractor squares with a top or bottom gap was presented.

\section{Results and discussion}

The same analysis as Experiment 2, a repeated-measures three-way ANOVA with singleton distractor, search difficulty, and search task as factors, was applied to the RT data. The RT analysis revealed significant main effects of singleton distractor, $F(1,19)=4.49, p<.048, \eta^{2}=.19$, and search difficulty, $F(1,19)=430.7, p<.001, \eta^{2}=.96$. While the main effect of search task was not found, $F(1,19)=1.987, p=.18$, the interaction between singleton distractor and search task was significant, $F(1,19)=13.24, p=.002, \eta^{2}=.41$. The two-way interactions between search task and search difficulty, $F(1,19)=192.8, p<.001, \eta^{2}=.91$, and search difficulty and singleton distractor, $F(1,19)=7.674, p=.012, \eta^{2}=.29$, were also significant. The three-way interaction was not significant $(p=.071)$.

As illustrated in Fig. 4, Experiment 3 replicated the results from Experiment 2 . When we separately examined the feature search and the Landolt- $\mathrm{C}$ search data, consistent with findings from Experiment 2, a color singleton distractor captured attention under the orientation-feature search, $F(1,19)=11.6, p=$ $.003, \eta^{2}=.38$. This capture increased when the search efficiency decreased, $F(1,19)=8.932, p=.008, \eta^{2}=.32$. On the contrary, a color singleton distractor did not capture attention under the Landolt-C search.

The three-way ANOVA was also applied to the accuracy data. The full details of the ANOVA results are presented in Table S4 in the Supplementary Material. The resulting mean accuracy rates are shown in Table 2. Search accuracy was high, with no difference between the distractor-present and distractor-absent trials $(p>.07)$. These results show that the current RT results were not contaminated by a speed-accuracy trade-off.

Experiment 3 confirmed the findings drawn from Experiments 1 and 2. Attentional capture by a singleton stimulus of the two tasks showed contrasting patterns - even when participants performed Landolt-C search task, in which a set of homogenous distractors was used. Further, the magnitude of this capture effect increased as search efficiency decreased under the feature search task. Considering all the results of Experiments 1, 2, and 3 (see Table S5 in the Supplementary Material), it is clear that search slope by itself cannot determine the presence/absence of attentional capture by the singleton distractor.

\section{General discussion}

The present study investigated how the cognitive mechanism underlying a visual search task affects stimulus-driven attentional capture. In three experiments, we found that while participants performed an orientation-feature search task, a salient, but task-irrelevant distractor captured attention, 
(a)

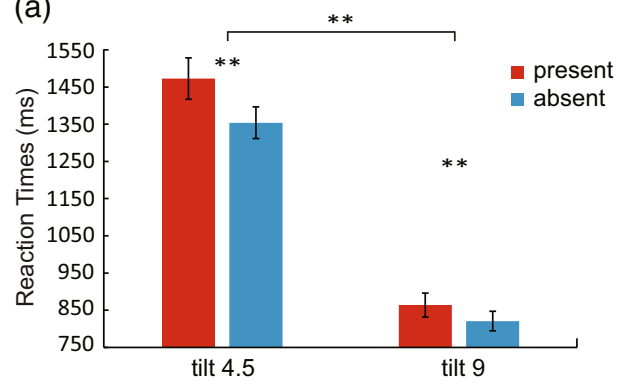

(b)

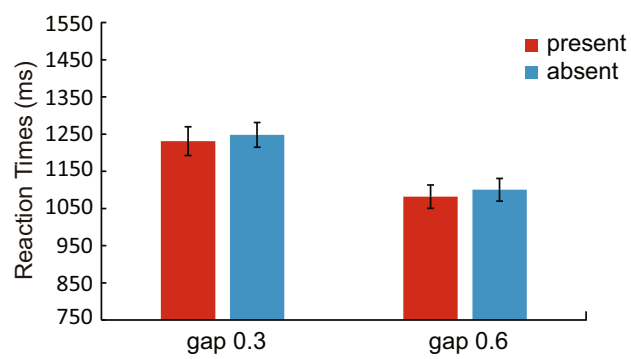

Fig. 4 Search RTs of Experiment 3. Error bars represent standard errors of the means. Mean reaction times of orientation-feature search task (a) and Landolt-C search task (b)

interfering with the search process. The magnitude of this capture effect increased as search efficiency decreased (Barras and Kerzel, 2017b). However, no stimulus-driven attentional capture was found under the Landolt-C search task.

Why was stimulus-driven attentional capture found only under the feature search but not under the Landolt-C search? To resolve this issue, we suggest considering the nature of given visual search tasks. To perform the Landolt-C search task, the limited capacity of attentional resource should be serially allocated to each item, taxing the attentional control system (Woodman \& Luck, 1999, 2003, 2007). By contrast, it is yet to be clarified how the inefficient feature search is performed. From the fact that the process of searching for a feature target, which slightly differs from distractors, yielded a robust set-size effect, it is tempting to conclude that the feature search is also performed in a serial manner. However, it is possible that multiple items are simultaneously processed with limited attentional resources allocated to each item. This limited parallel search can produce similar set-size effects as the serial search does.

Furthermore, growing evidence shows that the presence of statistical decision noise can also produce a significant set-size effect when a given search does not depend on capacitylimited attentional resources. Specifically, since neural representations of search items are inherently noisy, the probability that a nontarget item is confused with the target stimulus increases as the number of search items in the display increases (Eckstein, Thomas, Palmer, \& Shimozaki, 2000; Palmer, 1994; Palmer et al., 1993). With this statistical decision noise, even in the case where all the search items are simultaneously processed in a capacity-unlimited manner, search performance should suffer as the number of search items increases.

Supporting this, several recent studies showed that inefficient feature search tasks, yielding a robust set-size effect, can be performed without the recruitment of capacity-limited attentional resources (Han, 2017; Huang \& Pashler, 2005; Scharff, Palmer, \& Moore, 2011, 2013). In those studies, the search items appeared in two different ways: simultaneous and sequential presentation. In the former, all the search items were simultaneously presented, while in the latter, two subsets of the search items were sequentially presented. If a search recruits capacity-limited attentional resource, then the search performance should be better in the sequential presentation than in the simultaneous presentation because the amount of capacity allocated to each item at a given time is doubled by the sequential presentation. Importantly, the two conditions included the same number of search items, equating the total amount of statistical decision noise. Using this paradigm, it was found that the performance of feature searches did not benefit from the sequential presentation, even though the searches were inefficient (Han, 2017; Huang \& Pashler, 2005). These results indicate that the feature searches, however inefficiently they are performed, proceed without the recruitment of much attentional resource.

Given the above, while we admit that further research is needed to clarify the exact nature of the feature search process, extant evidence suggests that the performance of the Landolt$\mathrm{C}$ search task strains attentional control to a greater extent than the feature search does. Based upon this, the stimulus-driven capture of attention by a salient distractor seems to be heavily dependent on how the attentional control system is deployed. This claim is broadly consistent with the goal-driven theory of attentional capture proposed by Folk, Remington, and Johnston (1992). In their seminal study, in which a spatial cueing paradigm was employed, Folk and colleagues argued that a stimulus captures attention only when it matches a topdown task set. Specifically, they found that color singleton cues captured attention only when the colors of the cue and target matched. By contrast, a salient cue, whose color differed from the target color, did not capture attention. Similarly, Bacon and Egeth (1994) found that a salient singleton distractor captured attention in visual search only when participants adopted a strategy of detecting a singleton stimulus to locate the target (singleton-detection mode). By contrast, no capture was found when the strategy of searching for a singleton could not be used.

However, these accounts cannot easily accommodate recent findings that a distractor with distinct colors captured attention when stimulus color was a task-irrelevant feature. In the experiments by Barras and Kerzel (2017b) and the present experiments, participants searched for a shapedefined or an orientation-defined target. In this case, colors 
of the stimuli were not related to the task setting. Furthermore, the search tasks yielded significant set-size effects, implying that the target was not a pop-out stimulus. Hence, the singleton detection mode was not viable. Despite these, significant attentional capture by salient distractors was found. These results are inconsistent with the prediction from the strict goaldriven accounts of attentional capture.

Considering that the criterion determining whether a given search is an efficient or inefficient search is the search slope of $10 \mathrm{~ms} /$ item (Wolfe \& Horowitz, 2004), most of the visual searches in the present study should be classified as excessively inefficient searches; the search slopes of the feature search tasks ranged from $11 \mathrm{~ms} /$ item to $117 \mathrm{~ms} /$ item, while the Landolt-C search tasks yielded search slopes ranging from $68 \mathrm{~ms} /$ item to $109 \mathrm{~ms} /$ item. The fact that attentional capture by the salient distractor was found only under the feature search suggests that search efficiency cannot solely determine whether a salient stimulus captures attention or not.

Finally, the present findings provide support for the attentional window account suggested by Theeuwes (2004), which has been challenged by Barras and Kerzel (2017b). It has been presumed that efficient search is done in a parallel manner, setting a wide attentional window, while inefficient search proceeds in a serial manner, reducing the size of the attentional window. However, as mentioned above, there is evidence that some inefficient searches, such as the orientation-feature search of the present study, are indeed parallel searches, deploying a wide attentional window. Under this inefficient but parallel search, significant attentional capture was found. However, when search should be done in a serial manner, as in the Landolt-C search, no capture occurred. Hence, we suggest that the attentional window size account is still a viable account to reconcile conflicting results.

To conclude, the present study showed that a salient stimulus captured attention and affected target processing when participants performed an orientation-feature search task, whereas this capture was absent or minimized under the Landolt-C search. While search efficiency has been considered as a crucial factor to observe stimulus-driven attentional capture, we provide empirical evidence calling for the modification of the extant theoretical framework. Our results suggest that important factors of stimulus-driven attention in visual search are the nature of the search process and the extent to which attentional control is strained, as well as search efficiency.

Acknowledgements K. Jung and Y. Min were supported by the MSIT (Ministry of Science and ICT), Korea, under the ITRC (Information Technology Research Center) support program (IITP-2018-2016-000304) supervised by the IITP (Institute for Information \& Communications Technology Promotion).

S. W. Han was supported by the Ministry of Education of the Republic of Korea and the National Research Foundation of Korea (NRF2017S1A5A8018781).

\section{References}

Bacon, W. F., \& Egeth, H. E. (1994). Overriding stimulus-driven attentional capture. Perception \& Psychophysics, 55(5), 485-496.

Barras, C., \& Kerzel, D. (2017a). Salient-but-irrelevant stimuli cause attentional capture in difficult, but attentional suppression in easy visual search. Psychophysiology, 54(12), 1826-1838.

Barras, C., \& Kerzel, D. (2017b). Target-nontarget similarity decreases search efficiency and increases stimulus-driven control in visual search. Attention, Perception, \& Psychophysics, 79(7), 2037-2043. doi:https://doi.org/10.3758/s13414-017-1367-9

Beck, D. M., \& Kastner, S. (2005). Stimulus context modulates competition in human extrastriate cortex. Nature Neuroscience, 8(8), 1110.

Beck, D. M., \& Kastner, S. (2009). Top-down and bottom-up mechanisms in biasing competition in the human brain. Vision Research, 49(10), 1154-1165.

Desimone, R., \& Duncan, J. (1995). Neural mechanisms of selective visual attention. Annual Review of Neuroscience, 18(1), 193-222.

Eckstein, M. P., Thomas, J. P., Palmer, J., \& Shimozaki, S. S. (2000). A signal detection model predicts the effects of set size on visual search accuracy for feature, conjunction, triple conjunction, and disjunction displays. Perception \& Psychophysics, 62(3), 425-451.

Folk, C. L., Remington, R. W., \& Johnston, J. C. (1992). Involuntary covert orienting is contingent on attentional control settings. Journal of Experimental Psychology Human Perception and Performance, 18, 1030-1030.

Gaspelin, N., Ruthruff, E., \& Lien, M. C. (2016). The problem of latent attentional capture: Easy visual search conceals capture by taskirrelevant abrupt onsets. Journal of Experimental Psychology: Human Perception and Performance, 42(8), 1104-1120. doi: https://doi.org/10.1037/xhp0000214

Gaspelin, N., Ruthruff, E., Lien, M.-C., \& Jung, K. (2012). Breaking through the attentional window: Capture by abrupt onsets versus color singletons. Attention, Perception, \& Psychophysics, 74(7), 1461-1474.

Han, S. W. (2017). Search for capacity-limited and super-capacity search. Experimental Psychology, 64(3), 149.

Han, S. W., \& Marois, R. (2014). The effects of stimulus-driven competition and task set on involuntary attention. Journal of Vision, 14(7), $14-14$.

Huang, L., \& Pashler, H. (2005). Attention capacity and task difficulty in visual search. Cognition, 94(3), B101-B111.

Kastner, S., De Weerd, P., Desimone, R., \& Ungerleider, L. G. 1998. Mechanisms of directed attention in the human extrastriate cortex as revealed by functional MRI. Science, 282(5386), 108-111.

Kerzel, D., \& Barras, C. (2016). Distractor rejection in visual search breaks down with more than a single distractor feature. Journal of Experimental Psychology: Human Perception and Performance, 42(5), 648.

Lamy, D., \& Egeth, H. E. (2003). Attentional capture in singletondetection and feature-search modes. Journal of Experimental Psychology: Human Perception and Performance, 29(5), 1003.

Leber, A. B., \& Egeth, H. E. (2006). It's under control: Top-down search strategies can override attentional capture. Psychonomic Bulletin \& Review, 13(1), 132-138.

Mazyar, H., Van den Berg, R., \& Ma, W. J. (2012). Does precision decrease with set size? Journal of Vision, 12(6), 10-10.

McElree, B., \& Carrasco, M. (1999). The temporal dynamics of visual search: Evidence for parallel processing in feature and conjunction searches. Journal of Experimental Psychology: Human Perception and Performance, 25(6), 1517.

Palmer, J. (1994). Set-size effects in visual search: The effect of attention is independent of the stimulus for simple tasks. Vision Research, 34(13), 1703-1721. 
Palmer, J., Ames, C. T., \& Lindsey, D. T. (1993). Measuring the effect of attention on simple visual search. Journal of Experimental Psychology: Human Perception and Performance, 19(1), 108.

Palmer, J., Verghese, P., \& Pavel, M. (2000). The psychophysics of visual search. Vision Research, 40(10/12), 1227-1268.

Palmer, J., \& Wright, R. (1998). Attentional effects in visual search: Relating search accuracy and search time. Visual Attention, 8, 348-388.

Pavlov, I. P. (1927). Conditional reflexes: An investigation of the physiological activity of the cerebral cortex.

Peirce, J. W. (2007). PsychoPy-Psychophysics software in Python. Journal of Neuroscience Methods, 162(1), 8-13.

Proulx, M. J., \& Egeth, H. E. (2006). Target-nontarget similarity modulates stimulus-driven control in visual search. Psychonomic Bulletin \& Review, 13(3), 524-529.

Scharff, A., Palmer, J., \& Moore, C. M. (2011). Extending the simultaneous-sequential paradigm to measure perceptual capacity for features and words. Journal of Experimental Psychology: Human Perception and Performance, 37(3), 813.

Scharff, A., Palmer, J., \& Moore, C. M. (2013). Divided attention limits perception of 3-D object shapes. Journal of Vision, 13(2), 18-18.

Sokolov, E. N. (1963). Perception and the conditioned reflex. Oxford: Pergamon Press.

Sung, K. (2008). Serial and parallel attentive visual searches: Evidence from cumulative distribution functions of response times. Journal of
Experimental Psychology: Human Perception and Performance, 34(6), 1372.

Theeuwes, J. (1992). Perceptual selectivity for color and form. Attention, Perception, \& Psychophysics, 51(6), 599-606.

Theeuwes, J. (2004). Top-down search strategies cannot override attentional capture. Psychonomic Bulletin \& Review, 11(1), 65-70.

Treisman, A. M., \& Gelade, G. (1980). A feature-integration theory of attention. Cognitive Psychology, 12(1), 97-136.

Wolfe, J. M., \& Horowitz, T. S. (2004). What attributes guide the deployment of visual attention and how do they do it? Nature Reviews Neuroscience, 5(6), 495.

Woodman, G. F., \& Luck, S. J. (1999). Electrophysiological measurement of rapid shifts of attention during visual search. Nature, 400(6747), 867-869.

Woodman, G. F., \& Luck, S. J. (2003). Serial deployment of attention during visual search. Journal of Experimental Psychology: Human Perception and Performance, 29(1), 121.

Woodman, G. F., \& Luck, S. J. (2007). Do the contents of visual working memory automatically influence attentional selection during visual search? Journal of Experimental Psychology: Human Perception and Performance, 33(2), 363.

Woodman, G. F., Luck, S. J., \& Schall, J. D. (2007). The role of working memory representations in the control of attention. Cerebral Cortex, 17(Suppl. 1), i118-i124. 